\title{
Functional MRI, resting state fMRI, and DTI for predicting verbal fluency outcome following resective surgery for temporal lobe epilepsy
}

\author{
Karol Osipowicz, PhD, ${ }^{1,3}$ Michael R. Sperling, MD, ${ }^{1}$ Ashwini D. Sharan, MD, ${ }^{2}$ and \\ Joseph I. Tracy, PhD' \\ Departments of ${ }^{1}$ Neurology and ${ }^{2}$ Neurosurgery, Thomas Jefferson University/Sidney Kimmel Medical College; and ${ }^{3}$ Department \\ of Psychology, Drexel University, Philadelphia, Pennsylvania
}

\begin{abstract}
OBJECTIVE Predicting cognitive function following resective surgery remains an important clinical goal. Each MRI neuroimaging technique can potentially provide unique and distinct insight into changes that occur in the structural or functional organization of "at-risk" cognitive functions. The authors tested for the singular and combined power of 3 imaging techniques (functional MRI [fMRI], resting state fMRI, diffusion tensor imaging) to predict cognitive outcome following left (dominant) anterior temporal lobectomy for intractable epilepsy.

METHODS The authors calculated the degree of deviation from normal, determined the rate of change in this measure across the pre- and postsurgical imaging sessions, and then compared these measures for their ability to predict verbal fluency changes following surgery.

RESULTS The data show that the 3 neuroimaging techniques, in a combined model, can reliably predict cognitive outcome following anterior temporal lobectomy for medically intractable temporal lobe epilepsy.

CONCLUSIONS These findings suggest that these 3 imaging modalities can be used effectively, in an additive fashion, to predict functional reorganization and cognitive outcome following anterior temporal lobectomy.

http://thejns.org/doi/abs/10.3171/2014.9.JNS131422
\end{abstract}

KEY WORDS functional connectivity; white matter connectivity; fMRI; epilepsy; temporal lobe surgery; functional neurosurgery

$\mathrm{P}$ REDICTING outcome following resective brain surgery remains a high-priority goal in neurology and neurosurgery. Recently, a need for classifying surgical outcome on the basis of functional status, such as cognition, rather than just seizure control, has been articulated. ${ }^{44}$ A number of neuroimaging techniques give us insight into the neuroanatomical correlates of cognition. These techniques are increasingly being used as part of presurgical planning algorithms, ${ }^{11,19,20,28}$ providing an opportunity to not only gain insights into the functional neuroanatomy of "at-risk" cognition and how it relates to the underlying pathology, but also use such insights to predict cognitive outcome following surgery, such as anterior temporal lobectomy (ATL) for epilepsy.
The predictive power and the combined clinical utility of 3 major MRI techniques (functional MRI [fMRI], resting state fMRI [rsfMRI], and diffusion tensor imaging [DTI]) are unknown and untested. These 3 modalities can be conceptually combined to obtain a more complete view of the neural modules and connectivity networks implementing cognition and driving adaptive or maladaptive neuroplasticity responses in the brain. Functional MRI can be used to define an active network implementing a key function that might be "at risk" with temporal lobe surgery (e.g., verbal fluency), although the validity of this approach needs to be established in prospective studies. ${ }^{5}$ Preoperative fMRI has been shown to be sensitive to language and verbal memory laterality ${ }^{9}$ and is a known predictor of cog-

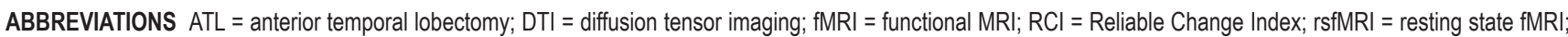
TLE = temporal lobe epilepsy.

SUBMITTED July 3, 2013. ACCEPTED September 3, 2014.

INCLUDE WHEN CITING Published online September 25, 2015; DOI: 10.3171/2014.9.JNS131422. 
nitive outcome ${ }^{24,45}$ in the setting of epilepsy. For instance, a large fMRI study of verbal and visual memory in patients undergoing left or right anterior temporal lobectomy suggested that presurgical fMRI activation of the ipsilateral anterior temporal lobe was correlated with greater postsurgical memory decline. ${ }^{6}$ Resting state fMRI can be used to capture correlated neural signaling, identifying the network of gray matter regions that are biased toward neural communication, although no research has yet evaluated its surgical applicability. ${ }^{37}$ DTI can be used to evaluate the integrity of and specify the anatomical connectivity between the functionally relevant gray matter regions. One published study has reported that a change in fractional anisotropy correlates with good language outcome following temporal lobectomy, but this study lacked a normal control group. ${ }^{45}$ In short, rsfMRI can identify the cortical regions communicating within a network; DTI can verify that these regions are anatomically connected; and fMRI can define the cognitive function(s) the network may be communicating about. In practical terms, all three can be gathered during one MRI scanning session with a minimal increase in scanning time and no increase in patient discomfort.

Despite the growing use of task-driven fMRI, DTI, and even resting state imaging for clinical purposes, our knowledge of the comparative sensitivity of each technique to cognitive and behavioral change following surgery is limited. Nor do we understand how to use these 3 imaging techniques in an integrated fashion within our presurgical algorithms as a means of enhancing surgical outcomes. More specifically, to date no empirical study has examined all 3 modalities for their single or joint power in predicting cognitive or seizure outcome following surgery. It is important to note that the most reliable current predictors of postsurgical cognitive outcome remain baseline neurocognitive testing, the intracarotid amobarbital examination, the surgical hemisphere (dominant, nondominant), and epilepsy duration, $7,10,17,25$ and no study has compared the predictive power of the emerging imaging modalities with some of the more established predictors.

In this report, we investigate the predictive power of these imaging modalities by calculating the degree of deviation from normal for each modality, focusing on a specific network involved in a key at-risk function, namely verbal fluency. The relative change in this deviation score from pre- to postsurgery is used to capture alterations and potential reorganization in the verbal fluency network following ATL. Although the role of the dominant mesial temporal lobe in expressive language is poorly understood, $, 2,8,13,16,33,34$ we chose verbal fluency as word-finding and expressive language difficulties are among the most common complaints made by patients after resective surgery.,16

Based on the unique information about brain networks offered by each imaging modality, we hypothesize that each will contribute important information about postsurgical cognitive outcome, making a combined, multimodal statistical model the most robust in terms of effectively predicting postsurgical cognitive outcome. We use fMRI to create a brain map of verbal fluency followed by characterizations of resting state functional connectivity and white matter anatomical connectivity utilizing the fMRI verbal fluency network as the seed. More specifically, we hypothesize that utilizing all 3 modalities will be superior at predicting verbal fluency outcome compared to any single imaging modality, as well as to established demographic and neuropsychological predictors. Finally, because we are focusing on verbal (semantic) fluency, we expect change in the fMRI networks to be the most predictive single modality, as this modality relies on a semantic fluency task as stimulation.

\section{Methods}

\section{Participants}

A total of 15 patients with left temporal lobe epilepsy were recruited from the Jefferson Comprehensive Epilepsy Center. A total of 20 age-matched healthy controls were recruited from the Thomas Jefferson University community. All patients underwent a standardized en bloc resection of the anterior temporal lobe, including amygdalohippocampectomy, as treatment for medically intractable temporal lobe epilepsy (TLE). Patients underwent neuroimaging before (mean 45 days) and after (mean 219 days) surgery. (For details of the Jefferson Comprehensive Epilepsy Center algorithm for surgical decision making see Sperling et al..$^{38}$ ) All patients met the following inclusion criteria: unilateral temporal lobe seizure onset (as localized through surface video/electroencephalography recordings); MRI and/or PET confirmation of epileptogenic temporal lobe pathology; and a Full Scale IQ of at least 75. All controls were free of any psychiatric or neurological disorders. The study was approved by the Institutional Review Board for Research with Human Subjects at Thomas Jefferson University, and all participants provided written informed consent. Table 1 outlines the demographic and clinical characteristics of the participants.

\section{Scanning Parameters}

All patients underwent MRI with a 3-T X-series Philips Achieva clinical MRI scanner using an 8-channel head coil during a 4-year period (2007-2011). There were no changes to the scanner software (reconstructer version 8), hardware, or sequence during that time. Participant data were collected in a pseudo-randomized, interleaved fashion (i.e., not all preoperative scans were completed before all postoperative scans), so as to not introduce any bias based on temporally dependent scanner calibration.

Each participant's data were acquired utilizing the following scan order: survey, field reference inhomogeneity scan, T1 (for details on T1 data acquisition, see Osipowicz et al. ${ }^{32}$ ), and experimental scans (fMRI, rsfMRI, and DTI). The experimental scans were collected in positions identical to and prescribed by the MPRage (anterior commissure-posterior commissure line). All data were collected and analyzed using standard and established methods. For details and the specific parameters related to collection and analysis of each image modality, see Osipowicz et al. ${ }^{32}$ and Tracy et al. ${ }^{40}$ and Supplemental Data.

\section{Neurocognitive Measure of Cognitive Outcome and Cognitive Task Network Definition Through fMRI}

Patients were classified according to cognitive outcome, 
TABLE 1. Summary of patient demographic and clinical characteristics

\begin{tabular}{|c|c|c|c|c|c|c|c|c|c|c|c|}
\hline \multirow[b]{2}{*}{ Case No. } & \multirow[b]{2}{*}{ Sex } & \multirow[b]{2}{*}{$\begin{array}{l}\text { Age } \\
(y r s)^{*}\end{array}$} & \multirow[b]{2}{*}{$\begin{array}{l}\text { Ep Dur } \\
\text { (yrs) }\end{array}$} & \multicolumn{2}{|c|}{ VFL } & \multirow[b]{2}{*}{$\begin{array}{l}\text { Cognitive } \\
\text { Outcome }\end{array}$} & \multirow[b]{2}{*}{ Path } & \multirow[b]{2}{*}{$\begin{array}{l}\text { Sz Freq/ } \\
\text { Mo }\end{array}$} & \multirow[b]{2}{*}{ Sz Type } & \multirow[b]{2}{*}{$\mathrm{EC} \ddagger$} & \multirow[b]{2}{*}{ AEDs } \\
\hline & & & & $\mathrm{BL}$ & Postop & & & & & & \\
\hline 1 & M & 26 & 21 & 24 & 32 & Good & Atrophy & 1 & CPS, SPS, CPS/G & $A$ & Levetiracetam, lamotrigine \\
\hline 2 & M & 28 & 12 & 19 & 12 & Poor & Enlarge (horn) & 2 & CPS, CPS/G & $A$ & Levetiracetam, oxcarbazepine \\
\hline 3 & $\mathrm{~F}$ & 41 & 36 & 11 & 3 & Poor & MTS & 16 & CPS, SPS & $\mathrm{C}$ & $\begin{array}{l}\text { Levetiracetam, valproic acid, } \\
\text { phenobarbital }\end{array}$ \\
\hline 4 & M & 30 & 11 & 21 & 20 & Good & MTS & 2 & CPS, GTC & $A$ & Levetiracetam \\
\hline 5 & $\mathrm{~F}$ & 50 & 31 & 21 & 13 & Poor & Atrophy & 7 & CPS, CPS/G & C & Phenytoin \\
\hline 6 & M & 47 & 42 & 21 & 11 & Poor & Hippo atrophy & 15 & CPS & $A$ & Carbamazepine, irbesartan \\
\hline 7 & $\mathrm{~F}$ & 42 & 4 & 18 & 8 & Poor & MTS & 1 & CPS & A & Lamotrigine, methimazole \\
\hline 8 & $\mathrm{~F}$ & 32 & 8 & 17 & 18 & Good & MTS & 1 & CPS, CPS/G & C & Lamotrigine, carbamazepine \\
\hline 9 & $\mathrm{~F}$ & 53 & 40 & 13 & 18 & Good & MTS & 5 & CPS, GTC & A & Lamotrigine, carbamazepine \\
\hline 10 & M & 65 & 45 & 16 & 20 & Good & Hippo atrophy & 7 & CPS & A & Topiramate, olanzapine \\
\hline 11 & $\mathrm{~F}$ & 39 & 26 & 16 & 21 & Good & Hippo atrophy & 1 & CPS & $A$ & $\begin{array}{l}\text { Valproic acid, phenobarbital, } \\
\text { carbamazepine }\end{array}$ \\
\hline 12 & $M$ & 60 & 56 & 18 & 11 & Poor & MTS & 2 & CPS, SPS & $A$ & Levetiracetam \\
\hline 13 & $\mathrm{~F}$ & 52 & 32 & 23 & 17 & Poor & Enlarge (horn) & 1 & CPS, GTC & $A$ & Lamotrigine, carbamazepine \\
\hline 14 & $\mathrm{~F}$ & 41 & 37 & 26 & 25 & Good & MTS & 2 & CPS, GTC & $A$ & $\begin{array}{l}\text { Venlafaxine, lamotrigine, } \\
\text { levetiracetam }\end{array}$ \\
\hline 15 & $\mathrm{~F}$ & 26 & 5 & 14 & 17 & Good & Atrophy & 20 & CPS & $A$ & $\begin{array}{l}\text { Diazepam, levetiracetam, } \\
\text { topiramate }\end{array}$ \\
\hline Mean (SD) & & $42(12)$ & $27(16)$ & $18(4)$ & $16(7)$ & & & $5(6)$ & & & \\
\hline
\end{tabular}

$\mathrm{AEDs}=$ antiepileptic drugs; $\mathrm{BL}=$ baseline; $\mathrm{CPS}=$ complex partial seizures; $\mathrm{CPS} / \mathrm{G}=$ complex partial seizures with secondary generalization; $\mathrm{EC}=$ postsurgical Engel Class I subscore; enlarge = enlargement; Ep Dur = epilepsy duration; GTC = generalized tonic-clonic seizures; Hippo = hippocampal; MTS = mesial temporal sclerosis; Path = temporal lobe pathology; SPS = simple partial seizures; $\mathrm{Sz}=$ seizure; VFL = verbal (semantic) fluency.

* Age at baseline scan.

$\dagger$ Based on RCl.

utilizing a verbal fluency measure collected as part of the neuropsychological assessment of language conducted on a presurgical (within 3 months of surgery) and postsurgical (1 year postsurgery) basis. The Controlled Oral Word Association Test subtest involving Semantic [animal] Fluency was administered under standard conditions and instructions. ${ }^{43}$ During the task, the participant must generate members of the category "four-legged animals" for 1 minute, with the total number generated used as the variable of interest. The task requires semantic processing and lexical access of common nouns. A Reliable Change Index $(\mathrm{RCI})^{7,17}$ was computed for this measure, revealing that a change in either direction of 4 raw score points on the semantic fluency measure reflected reliable change. In terms of neuropsychological outcome following brain surgery, evidence of no change or improvement in performance is generally considered to constitute a good outcome, as both patterns represent a preservation of the "at-risk" skill or function. Therefore, based on these RCI data, we defined 2 groups of cognitive outcome, capturing either a good (improved or unchanged performance on the Semantic Fluency measure) or a poor outcome A total of 8 of 15 patients obtained a good outcome, and 7 of 15 obtained a poor outcome.

A verb-generation task was used as task-driven stimulation during fMRI. This task requires semantic processing of a visually presented common noun and lexical ac- cess of verbs and action words associated with that noun (see supplemental material online for details on the verbgeneration task). Prior work has demonstrated that this task reliably establishes expressive language lateralization and shows activation in Broca's area.,12,23,31

\section{Definition of the Normal Control Templates for Quantifying Network Deviations in Left ATL Patients}

The above verb-generation task was administered to our sample of normal healthy controls. The group activation map based on statistical comparison of the verbgeneration condition with the visual control condition revealed activation in the middle frontal gyrus (BA47); bilateral medial frontal gyri extending superiorly to include the superior frontal gyri (BA6); left middle temporal gyrus (BA22) extending inferiorly to include the fusiform gyrus (BA37); and the right inferior frontal gyrus (BA44) (see Fig. 1). These areas of significant activation defined the normative functional network that served as a seed for subsequent rsfMRI and DTI analyses.

Analyses (see Supplemental Data for methods) of our normal control rsfMRI data using the above fMRI-defined verb-generation network as seed revealed a large degree of functional connectivity within the left hemisphere. The significantly correlated regions include the left inferior frontal gyrus, extending posteriorly to include pre- 


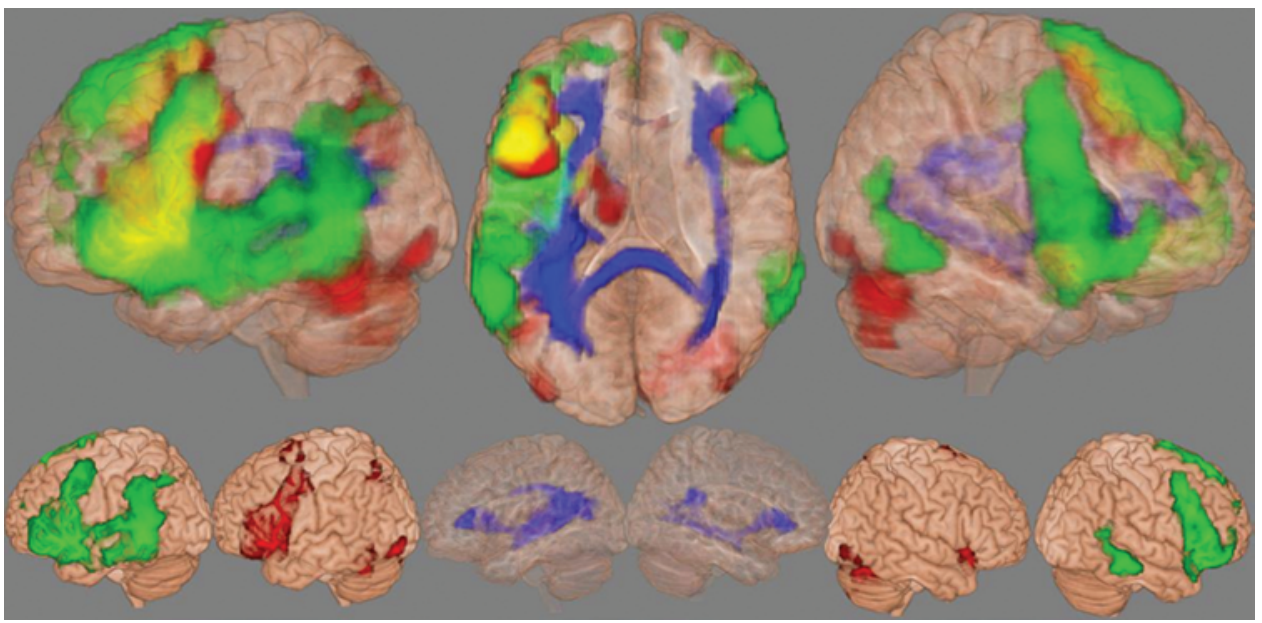

FIG. 1. The control templates (control group average) used for calculating deviations on fMRI (red), rsfMRI (green), and DTI (blue). Figure is available in color online only.

motor and primary motor areas and extending inferiorly to include the anterior temporal lobe. From the anterior temporal lobe this cluster extended posteriorly to include the posterior middle temporal gyrus, extending superiorly to include the superior temporal and supramarginal gyri. Also, bilateral medial frontal gyri functional connectivity was observed, quite similar to the bilateral medial frontal activations observed under fMRI. Lastly, in the right hemisphere, we saw a connectivity cluster that included the inferior frontal lobe and anterior and middle temporal lobes (see Fig. 1).

Using the same fMRI seeds for DTI tractography (see supplemental data online for methods), we saw white matter anatomical connectivity that involves the inferior longitudinal fasciculus, uncinate fasciculus, inferior fronto-occipital fasciculus, arcuate fasciculus, forceps major, corpus callosum (posterior splenium), and superior longitudinal fasciculus, all bilaterally. It should be noted that these tracks were more robust in the left hemisphere, with the left dominance most prominent in the forceps major and the superior longitudinal fasciculus (see Fig. 1).

\section{Deriving a Single Measure of Change From Pre- and Postsurgical Deviation Scores}

Methodological problems in measuring how to detect real versus random variation over time are important considerations for this type of project. To avoid problems we operationalize change by comparing each patient's imaging data with a modality-specific index of their deviation from normal at each point in time (presurgery, to be referred to as baseline, and postsurgery). This procedure provides a systematic means of quantifying the typicality of both pre- and postsurgical neurocognitive network organization, a procedure that alleviates concern about "double dipping" of analysis results..$^{21,26,42}$ Our methodology also minimizes reliance on random, session-specific fluctuations as part of the reference or comparison point for determining change.

For each imaging modality, we used the average of the maps for the normal controls (fMRI activation, rsfMRI connectivity, DTI tracts) as a template of the normal net- work and computed the goodness of fit between this template and each patient's individual map. We replicated the template-matching procedure used by Gross et al. ${ }^{14}$ In short, this is a nonlinear procedure that takes the number of the patient's voxels falling within the template relative to the total number of voxels in the template and subtracts the number of the patient's voxels falling outside of the template relative to the total number of brain voxels, yielding a goodness of fit for each individual map (Fig. 2). It should be noted that the type of score present in each voxel varies

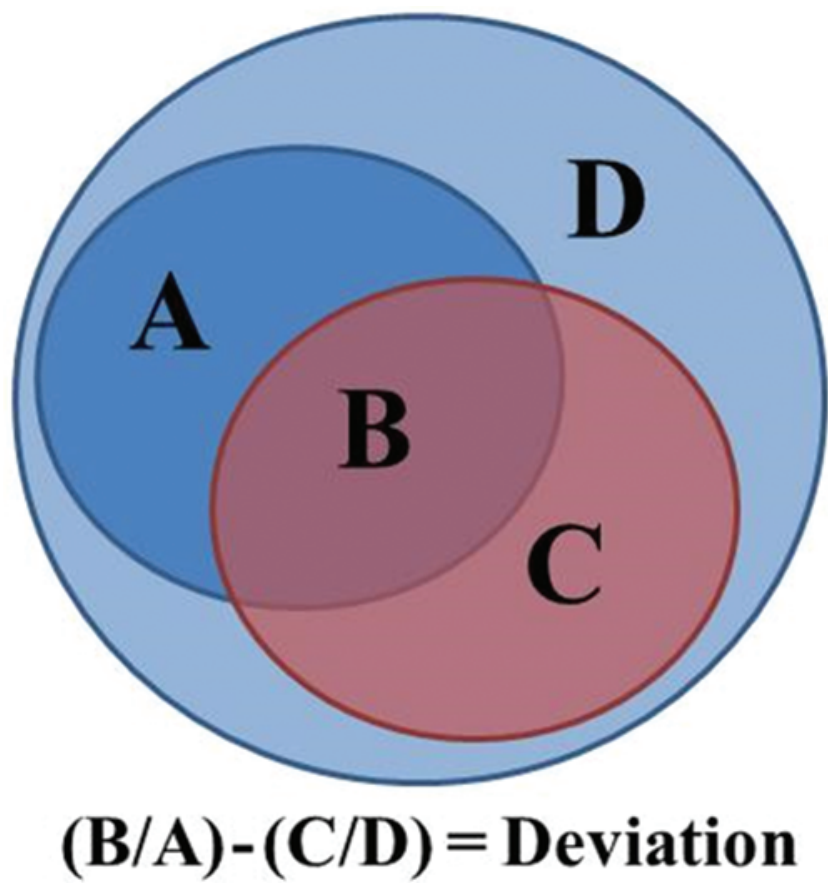

FIG. 2. Calculation of the deviation measure: A represents the number of voxels in the relevant template; $D$ represents the number of voxels in the brain absent the template (therefore, total brain volume equals $\mathrm{A}+$ $D) ; B$ represents the number of the patient's voxels that fall within the template (i.e., overlap between patient and template); $\mathrm{C}$ represents the number of the patient's voxels that do not fall within the template. Figure is available in color online only. 
for the different types of maps. For instance, fMRI utilizes a t-score, rsfMRI a Z-score, and DTI an inclusion variable. In interpreting the goodness-of-fit index, a score of 1.0 indicates a perfect fit with the normative template (all voxels within the template and none outside), and a score of zero indicates a poor fit (no voxels within the template; all outside the template) (See Fig. 3). For the logistic regression, once the goodness of fit is computed, this is transformed to a normalized deviation and expressed as a normal Z-score.

The change in this deviation score between the postand presurgical sessions was computed (i.e., postsurgical goodness of fit minus presurgical goodness of fit; referred to as the change score). For each modality, the closer the change score is to zero, the less change is observed across the 2 scanning sessions.

\section{Statistical Analyses}

To predict cognitive outcome, we built logistical regression statistical models comparing the change measures for each modality for their ability to reliably distinguish between good and poor cognitive outcome. We also assessed the predictive power of the baseline (presurgical) deviation of each of the imaging modalities. As a second level of analyses, we reexamined the ability of any significant predictors yielded by the above analyses for their ability to outperform rival hypotheses in terms of predictive power. These rival hypotheses included baseline measures of neuropsychological function (baseline semantic fluency), demographic variables (age, baseline Verbal IQ), and clinical measures representing important characteristics of epilepsy. All results reported obtained an alpha ( $p$ value) of $\leq 0.05$.

\section{Results}

Table 1 displays the clinical and demographic data for all participants. There was no observable difference between the groups on any of the variables listed, as assessed by Student t-test or chi-square test, as appropriate. Figure 3 displays the goodness-of-fit index for all 3 imaging modalities for the good and poor outcome groups at baseline (presurgery) and postsurgery.

\section{Comparing the Imaging Modalities for Their Ability to Predict Cognitive Outcome}

In the logistic regression (unique sum of squares) on

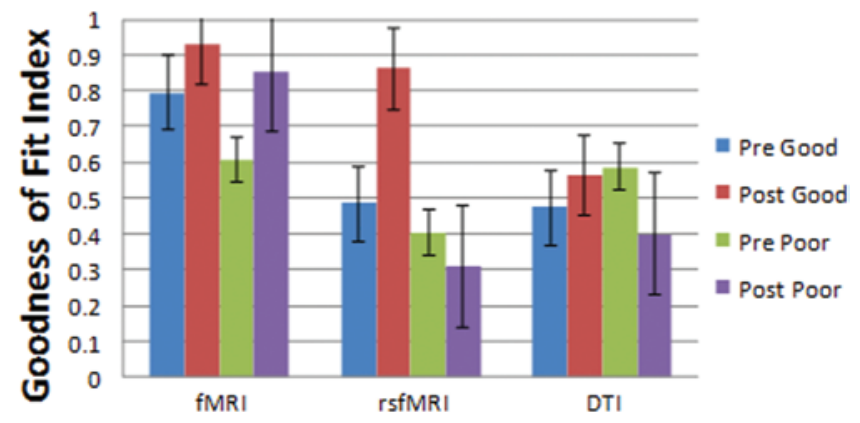

FIG. 3. Mean goodness of fit and standard error of each modality-specific deviation for the patients with good and poor outcomes, before (pre) and after (post) surgery. Figure is available in color online only. cognitive outcome containing the change scores of all 3 modalities, this 3 -variable model was significant (see Table 2 and Fig. 4). The model explained 52\% of the variance in outcome and correctly classified $87 \%$ of the patients. While no single effect in the model was significant, the standardized beta values suggested that DTI may have contributed the most in terms of predictive power. A classification plot (see Fig. 4) shows the good spread between good and poor outcome provided by the 3-modality predictive model.

To investigate the effect each of the change measures had on the model, we computed the incremental $\mathrm{R}^{2}$ for each change measure. This showed that DTI accounted for $32 \%$; resting state for $15 \%$; and fMRI for $4 \%$ of the observed variance. While no single-variable model significantly predicted outcome, the 2-variable model involving rsfMRI and DTI was significant, accounting for $49 \%$ of the variance in outcome.

We examined the 3 baseline deviation scores in a separate logistic regression model (unique sum of squares) of cognitive outcome. Here, the 3-variable model produced a statistical trend $(p<0.075)$, with $37 \%$ of the variance in outcome explained and $73 \%$ of the patients correctly classified on outcome. No single effect in the model was significant, although the standardized beta values suggested that baseline DTI deviation may have contributed the most in terms of predictive power.

Next, we sought to determine whether the 3-variable model involving change scores was a better predictor of cognitive outcome after accounting for the effects of the baseline imaging data. The 3-variable logistic regression was rerun using each modality's baseline (presurgical) deviation score as a covariate, with separate logistic regressions for each covariate. In each case, the 3-variable model remained significant, with the variance explained comparable to the original logistic regressions $(51.9 \%$ for the original model; $52.3 \%$ for the model with the fMRI baseline; $52.1 \%$ with the rsfMRI baseline; $52.3 \%$ with the DTI baseline). Again, among the predictor variables, no standardized beta was significant. In no case did the standardized beta value for baseline deviation score exceed that of the change scores, indicating that after accounting for these baseline covariates, the original 3-variable model containing the change scores for all 3 imaging modalities remained significant.

TABLE 2. Results of the main logistical regression models

\begin{tabular}{lccc}
\hline \multirow{2}{*}{ Result } & \multicolumn{3}{c}{ Model } \\
\cline { 2 - 4 } & Change Score & Baseline & $\begin{array}{c}\text { Change \& } \\
\text { Baseline }\end{array}$ \\
\hline Model significance & 0.012 & 0.075 & 0.002 \\
\hline Chi-square & 10.96 & 6.89 & 20.7 \\
\hline Cox \& Snell R & 0.519 & 0.369 & 0.749 \\
\hline$\%$ correct classification & 86.7 & 73.3 & 100 \\
\hline Odds ratio & 1.4 & 1.63 & $-\dagger$ \\
\hline Strongest predictor* & DTI $(-1.74)$ & DTI $(2.1)$ & - \\
\hline
\end{tabular}

* The strongest predictor is noted with its beta weight in parentheses.

$\dagger$ Because there was no error in correct classification, the odds ratio and strongest predictor could not be estimated for the model containing both change and baseline deviations. 


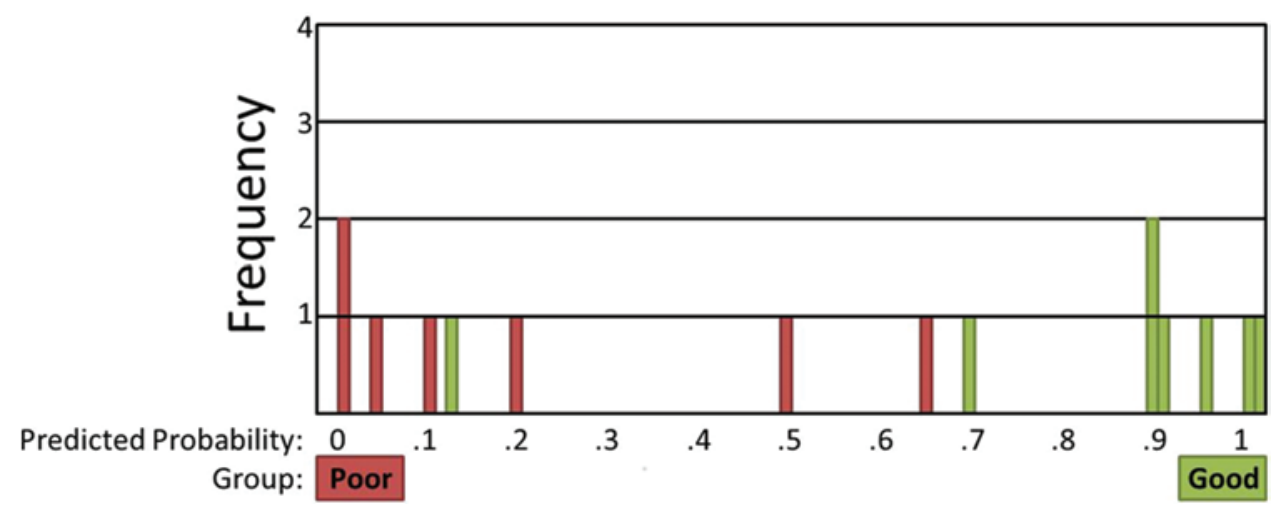

FIG. 4. Classification plot for the key 3-modality logistical regression model. Figure is available in color online only.

Lastly, to better understand the strength of the above 3 -modality model in predicting cognitive outcome, we examined demographic and clinical measures to determine if they constitute rival or alternate explanatory factors. Similar to the logistic regression analysis with the baseline deviation scores, we reran the 3-variable logistic regression model, in separate analyses, with each of the above measures inserted as a single covariate. The specific covariates tested were epilepsy duration, age, baseline animal naming score, and baseline Verbal IQ. For each covariate, the 3-variable logistic regression model remained significant with the proportion of variance explained in cognitive outcome comparable to the initial model $(52.7 \%$ for model with epilepsy duration; $52.9 \%$ with age; $59.3 \%$ with animal naming, $56.7 \%$ with Verbal IQ). In no case did the standardized beta value for covariate exceed that of the change scores, indicating that after accounting for these demographic and clinical measures, the original 3 -variable model containing the change scores remained significant.

\section{Discussion}

The purpose of this study was to test whether the changes observed on 3 brain mapping modalities are predictive of postsurgical cognitive outcome, specifically verbal fluency, following dominant-side ATL for intractable TLE. The data show that a statistical model combining 3 change variables, one from each modality, reliably predicts verbal fluency outcome, outperforming any model containing change scores from any single or duple pairing of the modalities. We found that a 2-variable model with rsfMRI and DTI (with fMRI excluded) also produced a statistically significant model of prediction. However, as was the case with the 3-variable model, no individual modality was predictive of outcome. In the 3-variable model, DTI accounts for the largest single amount of observed variance in verbal fluency outcome.

The direction of change scores indicated that for the patients with good outcomes, all 3 imaging modalities displayed a better match to the normative template postsurgery, suggesting that normalization had occurred (see goodness-of-fit data, Fig. 3). In quantitative terms, the degree of fit relative to the normative template changed most dramatically for the resting state functional connectivity measure. This pattern suggests that achieving a good out- come involves engaging normative regions to complete the task, regions that were previously less involved. Yet, to do so appears to require achieving more normative forms of connectivity for both gray and white matter. This indicates that when the anterior and mesial temporal lobe are absent, supporting prior or improved levels of performance requires a new, more typical patterning of white and gray matter connectivity.

In contrast, for the patients with poor outcomes the pattern of change across the 3 modalities was mixed. The task-fMRI measure showed a shift toward the template, a better fit postsurgery, but the resting state and DTI measures shifted away from the template. Our data suggest that good cognitive outcomes, even in the setting of more normative fMRI activation, require changes in functional and white matter connectivity before cognitive change can be effected. Gray and white matter connectivity, less normative in our poor-outcome patients, may not have been providing sufficient scaffolding to implement the neural communication necessary for the task. Said differently, the regional reorganization to implement better verbal fluency was present postsurgery, but the neuronal networks to support communication within the verbal fluency network were not in place. As to whether these resting state and white matter networks would, with time, reorganize, normalize, and come to implement a good verbal fluency outcome remains an open question. An intriguing possibility is that connectivity and task-related fMRI activations are on different trajectories postsurgery, reorganizing at different rates in good- and poor-outcome patients. Both the poor- and good-outcome groups were scanned at a mean interval of approximately 5 months postsurgery, but the poor-outcome group may have needed more time to recovery. For instance, the good-outcome group may recover and reorganize rapidly, implementing the task with the same areas seen in controls, areas that have renewed health as they have been unburdened by the impact of seizures. The poor-outcome group may be on a slower or less-effective trajectory, perhaps because changes in functional and white matter connectivity are hampered by the now-missing temporal lobe in ways that they are not in the good-outcome group. We should note that we sought to uncover meaningful group differences between the poor- and good-outcome groups in hopes of establishing the clinical features that might support their very differ- 
ent imaging patterns postsurgery, but we observed no reliable group differences. Another possibility is that the more aberrant connectivity characteristics of the poor-outcome group postsurgery suggest that these patients did not have the reserve capacity to reorganize network connectivity and normalize cognitive performance in the face of ATL.

Importantly, we found that the model containing the baseline (presurgical) measures from the 3 modalities was a weak and nonsignificant predictor of cognitive outcome. The combined 3-modality model of change remained significant even after accounting for these baseline measures. We also found that the key 3-modality model of change was a superior predictor of verbal fluency when competing against established predictors of cognitive outcome ${ }^{35}$ such as baseline neuropsychological measures of verbal fluency or IQ, as well as demographic and clinical measures. The 3-modality model of change remained significant regardless of whether these rival hypotheses were examined singly or together against the 3-variable model. This is important, as it demonstrates that these neuroimaging modalities have added value compared with extant models of predication.

Our data also make it clear that verbal (semantic) fluency is an at-risk function in patients with left temporal lobe epilepsy, as a significant proportion of our patients (roughly half of the sample) did experience a decline in this skill despite having a good surgical outcome in terms of seizure control (n.b., all of our patients were seizure free postsurgery). Our data show that what best distinguished the good- from the poor-outcome patients was the change profile, not the baseline status, of their neuroimaging. While change was key, and superior to the baseline findings, there was still a statistical trend suggesting that with larger samples baseline neuroimaging characteristics may also become a reliable predictor. Such data suggest that presurgical deviation from normal (a measure of reorganization in response to pathology) has the potential to become an important index of potential cognitive outcome.

We were surprised to find that fMRI deviation was not a significant predictor of cognitive outcome, as we had initially hypothesized, and that the model containing only rsfMRI and DTI was a significant predictor of cognitive outcome. We believe this may be caused by 2 factors. Both rsfMRI and DTI are measures of connectivity, and it would make sense that changes in connectivity might be more sensitive to reorganization than a measure more sensitive to the nodes in the network arising from the implementation of a specific task. Said differently, rsfMRI and DTI may capture network changes that are part of a more general reorganization of language, with the assumption that it may be more parsimonious for the brain to reorganize a network to preserve functions for a wide range of tasks, rather than link the reorganization to a single task. In this sense, our fMRI results may be too task specific. Perhaps utilizing a different language measure or multiple language tasks would increase the strength of the relationship between fMRI and cognitive outcome. We used a verb-generation task because it is a reliable language-lateralization task, ${ }^{36}$ and because we have observed deficits in semantic fluency in TLE patients. ${ }^{39}$ Perhaps an fMRI task that is designed to specifically activate the anterior temporal lobe, such as the task described by Binder et al. ${ }^{5}$ and Hamberger et al. ${ }^{16}$ would provide change scores that are predictive of verbal fluency. It is important to note that since our analyses focused on networks defined by fMRI seeds, even though fMRI was not the best predictor, it is still necessary to define the other networks.

These results support our original hypothesis that a multimodal predictive model would be superior to any single modality in predicting verbal fluency outcome. This suggests that cognitive reorganization occurred in our ATL patients, with each modality capturing a unique aspect of that reorganization, compelling and recommending the use of all 3 modalities when trying to obtain the most reliable index of change. Importantly, our method quantified the deviation of the neural connectivity and the functional and anatomical neural networks of our patients from normal, examining the relative difference in those deviations as our index of change. In so doing, we avoided the use of our presurgical neuroimaging results as the template for comparing and determining change, minimizing concerns of double dipping of analysis results.

Our data indicate that ATL does reorganize networks. Prior research has demonstrated that TLE with an early age of onset ${ }^{15,16,30}$ or long duration ${ }^{4}$ can reorganize the neural representation of language. Ours is the first report that ATL, in the setting of a good seizure outcome, also results in a change in the neural representation of language evident both in terms of regional activation and the functional and white matter connectivity between these regions. Furthermore, our data also make clear that removal of the mesial temporal lobe alters language functioning and underlying language networks. Prior neuropsychological studies of language outcome following ATL have shown mixed results, with some showing a persistence of deficits, ${ }^{22}$ and others showing normalization..$^{27,29}$ A prior neuroimaging study investigating the effect of ATL on language reorganization $^{41}$ demonstrated the potential negative impact on language network organization (see also Kim et al..$^{18}$ ). None of the above studies, however, presents evidence of reorganization from other imaging modalities. Thus, ours is the first study to demonstrate that fMRI language network reorganization co-occurs with functional connectivity changes on resting state fMRI and white matter changes on DTI. We must emphasize again, however, that our data make clear that what most reliably distinguishes good- versus poor-outcome patients is the change and reorganization observed on all 3 levels of network analysis.

There are a number of study limitations that need to be addressed. Our study suffered from a small sample size, limiting power, perhaps making it more impressive that we were still able to predict cognitive outcome. Although we focused on cognitive outcome, we had also hoped to report the ability of our 3 imaging modalities to predict seizure outcome, the ultimate test of the success of an ATL. However, we observed no variability in seizure outcome-all patients in our sample had a good outcome at the point of our assessment.

To our knowledge, this is the first study to examine the power of 3 neuroimaging techniques for predicting cognitive outcome. Our data suggest that by calculating the presurgical deviation (from controls) of results of 3 neu- 
roimaging techniques we can predict cognitive outcome following ATL for medically intractable TLE. We propose that the best predictive models are those that contain rsfMRI, DTI, and fMRI. Functional MRI is necessary to generate the seeds for resting state networks and DTI tracts, and then using all 3 modalities synergistically is the best way to predict postsurgical cognitive outcome. Future research should investigate whether other language tasks, or tasks from other cognitive domains such as memory, improve or alter the relative or combined predictive power of the 3 modalities.

\section{Acknowledgment}

This work was supported, in part, by a grant from the National Institute for Neurological Disorders and Stroke (grant no. R21 NS056071-01A1) to Dr. Joseph I. Tracy.

\section{References}

1. Afzali M, Soltanian-Zadeh H, Elisevich KV: Tract based spatial statistical analysis and voxel based morphometry of diffusion indices in temporal lobe epilepsy. Comput Biol Med 41:1082-1091, 2011

2. Agosta F, Viskontas IV, Gorno-Tempini ML: FMRI of memory. Neuromethods 41:379-409, 2009

3. Bartha L, Mariën P, Brenneis C, Trieb T, Kremser C, Ortler $\mathrm{M}$, et al: Hippocampal formation involvement in a languageactivation task in patients with mesial temporal lobe epilepsy. Epilepsia 46:1754-1763, 2005

4. Bernhardt BC, Chen Z, He Y, Evans AC, Bernasconi N: Graph-theoretical analysis reveals disrupted small-world organization of cortical thickness correlation networks in temporal lobe epilepsy. Cereb Cortex 21:2147-2157, 2011

5. Binder JR, Gross WL, Allendorfer JB, Bonilha L, Chapin J, Edwards JC, et al: Mapping anterior temporal lobe language areas with fMRI: a multicenter normative study. Neuroimage 54:1465-1475, 2011

6. Bonelli SB, Powell RHW, Yogarajah M, Samson RS, Symms MR, Thompson PJ, et al: Imaging memory in temporal lobe epilepsy: predicting the effects of temporal lobe resection. Brain 133:1186-1199, 2010

7. Chelune GJ, Naugle RI, Lüders H, Sedlak J, Awad IA: Individual change after epilepsy surgery: practice effects and base-rate information. Neuropsychology 7:41-52, 1993

8. Cohen NJ, Ryan J, Hunt C, Romine L, Wszalek T, Nash C: Hippocampal system and declarative (relational) memory: summarizing the data from functional neuroimaging studies. Hippocampus 9:83-98, 1999

9. Concha L, Beaulieu C, Collins DL, Gross DW: White-matter diffusion abnormalities in temporal-lobe epilepsy with and without mesial temporal sclerosis. J Neurol Neurosurg Psychiatry 80:312-319, 2009

10. Concha L, Beaulieu C, Wheatley BM, Gross DW: Bilateral white matter diffusion changes persist after epilepsy surgery. Epilepsia 48:931-940, 2007

11. De Ribaupierre S, Fohlen M, Bulteau C, Dorfmüller G, Delalande O, Dulac O, et al: Presurgical language mapping in children with epilepsy: Clinical usefulness of functional magnetic resonance imaging for the planning of cortical stimulation. Epilepsia 53:67-78, 2011

12. Diniz PB, Salmon CE, Velasco TR, Sakamoto AC, Leite JP, Santos AC: White matter alterations in temporal lobe epilepsy. Proc SPIE 7965:796527, 2011

13. Dolan RJ, Fletcher PC: Dissociating prefrontal and hippocampal function in episodic memory encoding. Nature 388:582-585, 1997

14. Gross DW, Concha L, Beaulieu C: Extratemporal white matter abnormalities in mesial temporal lobe epilepsy dem- onstrated with diffusion tensor imaging. Epilepsia 47:13601363,2006

15. Hamberger MJ, Cole J: Language organization and reorganization in epilepsy. Neuropsychol Rev 21:240-251, 2011

16. Hamberger MJ, Seidel WT, Goodman RR, Williams A, Perrine K, Devinsky O, et al: Evidence for cortical reorganization of language in patients with hippocampal sclerosis. Brain 130:2942-2950, 2007

17. Hermann BP, Seidenberg M, Schoenfeld J, Peterson J, Leveroni C, Wyler AR: Empirical techniques for determining the reliability, magnitude, and pattern of neuropsychological change after epilepsy surgery. Epilepsia 37:942-950, 1996

18. Kim JH, Lee JM, Kang E, Kim JS, Song IC, Chung CK: Functional reorganization associated with semantic language processing in temporal lobe epilepsy patients after anterior temporal lobectomy: a longitudinal functional magnetic resonance image study. J Korean Neurosurg Soc 47:17-25, 2010

19. Knake S, Salat DH, Halgren E, Halko MA, Greve DN, Grant PE: Changes in white matter microstructure in patients with TLE and hippocampal sclerosis. Epileptic Disord 11:244250, 2009

20. Kozák LR, Tóth V, Barsi P, Rudas G: [Functional magnetic resonance imaging for cortical mapping in epilepsy.] Ideggyogy Sz 64:294-299, 2011 (Hungarian)

21. Kriegeskorte N, Simmons WK, Bellgowan PS, Baker CI: Circular analysis in systems neuroscience: the dangers of double dipping. Nat Neurosci 12:535-540, 2009

22. Langfitt JT, Rausch R: Word-finding deficits persist after left anterotemporal lobectomy. Arch Neurol 53:72-76, 1996

23. Li H, Xue Z, Dulay MF, Verma A, Wong S, Karmonik C, et al: Distinguishing left or right temporal lobe epilepsy from controls using fractional anisotropy asymmetry analysis. Lecture Notes Comput Sci 6326:219-227, 2010

24. Li Y, Du H, Xie B, Wu N, Wang J, Wu G, et al: Cerebellum abnormalities in idiopathic generalized epilepsy with generalized tonic-clonic seizures revealed by diffusion tensor imaging. PLoS One 5:e15219, 2010

25. Londoño A, Castillo M, Lee YZ, Smith JK: Apparent diffusion coefficient measurements in the hippocampi in patients with temporal lobe seizures. AJNR Am J Neuroradiol 24:1582-1586, 2003

26. Luna B, Velanova K, Geier CF: Methodological approaches in developmental neuroimaging studies. Hum Brain Mapp 31:863-871, 2010

27. Lutz MT, Clusmann H, Elger CE, Schramm J, Helmstaedter C: Neuropsychological outcome after selective amygdalohippocampectomy with transsylvian versus transcortical approach: a randomized prospective clinical trial of surgery for temporal lobe epilepsy. Epilepsia 45:809-816, 2004

28. Mahdavi A, Houshmand S, Oghabian MA, Zarei M, Mahdavi A, Shoar MH, et al: Developing optimized fMRI protocol for clinical use: comparison of different language paradigms. J Magn Reson Imaging 34:413-419, 2011

29. Martin RC, Sawrie SM, Edwards R, Roth DL, Faught E, Kuzniecky RI, et al: Investigation of executive function change following anterior temporal lobectomy: selective normalization of verbal fluency. Neuropsychology 14:501-508, 2000

30. Mbwana J, Berl MM, Ritzl EK, Rosenberger L, Mayo J, Weinstein S, et al: Limitations to plasticity of language network reorganization in localization related epilepsy. Brain 132:347-356, 2009

31. Nguyen D, Vargas MI, Khaw N, Seeck M, Delavelle J, Lovblad KO, et al: Diffusion tensor imaging analysis with tract-based spatial statistics of the white matter abnormalities after epilepsy surgery. Epilepsy Res 94:189-197, 2011

32. Osipowicz K, Rickards T, Shah A, Sharan A, Sperling M, Kahn W, et al: A test of the role of the medial temporal lobe in single-word decoding. Neuroimage 54:1455-1464, 2011 
33. Patterson K, Nestor PJ, Rogers TT: Where do you know what you know? The representation of semantic knowledge in the human brain. Nat Rev Neurosci 8:976-987, 2007

34. Patterson MD, Bly BM, Porcelli AJ, Rypma B: Visual working memory for global, object, and part-based information. Mem Cognit 35:738-751, 2007

35. Potter JL, Schefft BK, Beebe DW, Howe SR, Yeh HS, Privitera MD: Presurgical neuropsychological testing predicts cognitive and seizure outcomes after anterior temporal lobectomy. Epilepsy Behav 16:246-253, 2009

36. Powell HWR, Parker GJM, Alexander DC, Symms MR, Boulby PA, Wheeler-Kingshott CAM, et al: Abnormalities of language networks in temporal lobe epilepsy. Neuroimage 36:209-221, 2007

37. Schoene-Bake JC, Faber J, Trautner P, Kaaden S, Tittgemeyer $M$, Elger CE, et al: Widespread affections of large fiber tracts in postoperative temporal lobe epilepsy. Neuroimage 46:569-576, 2009

38. Sperling MR, O'Connor MJ, Saykin AJ, Phillips CA, Morrell $\mathrm{MJ}$, Bridgman PA, et al: A noninvasive protocol for anterior temporal lobectomy. Neurology 42:416-422, 1992

39. Thivard L, Lehéricy S, Krainik A, Adam C, Dormont D, Chiras J, et al: Diffusion tensor imaging in medial temporal lobe epilepsy with hippocampal sclerosis. Neuroimage 28:682-690, 2005

40. Tracy JI, Osipowicz K, Spechler P, Sharan A, Skidmore C, Doucet G, et al: Functional connectivity evidence of corticocortico inhibition in temporal lobe epilepsy. Hum Brain Mapp 35:353-366, 2014

41. van Turennout M, Ellmore T, Martin A: Long-lasting cortical plasticity in the object naming system. Nat Neurosci 3:1329_ 1334,2000

42. Viviani R: Unbiased ROI selection in neuroimaging studies of individual differences. Neuroimage 50:184-189, 2010

43. Wieshmann UC, Clark CA, Symms MR, Barker GJ, Birnie KD, Shorvon SD: Water diffusion in the human hippocampus in epilepsy. Magn Reson Imaging 17:29-36, 1999

44. Wrench JM, Matsumoto R, Inoue Y, Wilson SJ: Current chal- lenges in the practice of epilepsy surgery. Epilepsy Behav 22:23-31, 2011

45. Yogarajah M, Focke NK, Bonelli SB, Thompson P, Vollmar C, McEvoy AW, et al: The structural plasticity of white matter networks following anterior temporal lobe resection. Brain 133:2348-2364, 2010

\section{Disclosure}

Dr. Sharan reports an ownership interest in ICVRX and ICP and a consultant relationship with Medtronic and SJM. Dr. Sperling reports a consultant relationship with Accorda Therapeutics and electroCore.

\section{Author Contributions}

Conception and design: all authors. Acquisition of data: Osipowicz, Sharan, Tracy. Analysis and interpretation of data: Osipowicz, Sperling, Tracy. Drafting the article: Osipowicz, Tracy. Critically revising the article: Osipowicz, Tracy. Reviewed submitted version of manuscript: Osipowicz, Tracy. Approved the final version of the manuscript on behalf of all authors: Osipowicz. Statistical analysis: Osipowicz, Tracy. Administrative/technical/material support: all authors. Study supervision: Osipowicz, Tracy.

\section{Supplemental Information Online-Only Content}

Supplemental material is available with the online version of the article.

Supplemental Data. http://thejns.org/doi/suppl/10.3171/ 2014.9.JNS131422.

\section{Correspondence}

Karol Osipowicz, Department of Psychology, Drexel University, 276 Stratton Hall, 3521 Chestnut St., Philadelphia, PA 19104. email:dr.o@drexel.edu. 